\title{
USING DEEP LEARNING TO IDENTIFY AND CLASSIFY SUNSPOTS IN MAGNETOGRAMS
}

\author{
Letícia S. de Oliveira*, André Leon S. Gradvohl.
}

\begin{abstract}
Some of the solar activities, such as solar flares, release large amounts of radiation and energy that impact on Earth's life and technological systems. These flares usually come from sunspots, which derive from solar magnetic activities. Currently, some solar data allow predicting when a solar flare will occur. This paper reports the use of the Deep Learning technique to identify and classify sunspots using solar magnetograms automatically. Our results show an accuracy greater than $80 \%$.
\end{abstract}

\section{Key words:}

Solar flares, Deep Learning, Object Detection.

\section{Introduction}

The Sun produces solar flares by the breakdown of magnetic fields. Sunspots concentrate on these fields because they have a significant magnet activity. This work used a Deep Learning network (DL) to train models capable of automatically identify and classify sunspots in magnetograms, i.e., images that show the variation of magnetic fields on the solar photosphere.

To identify and classify the magnetograms, we created two datasets. In the beginning, we build a magnetograms database from Gradvohl[1], which we manually labeled 1000 images, pointing where are the sunspots on the image. After that, we created a new database just with the sunspots, containing a classification according to Helioviewer. The datasets are the basis of the Object Detection and Classification models.

After the two databases are ready, we adapted the data to use in the DIGITS software[2], which uses DL to train and test artificial neural networks. We used the models created with DIGITS to implement a program in Python.

\section{Results and Discussion}

Using DIGITS we made an Object Detection and a Classification models, both using Caffe, a DL framework. We tried many combinations of the models and the databases, looking for the best result.

We registered all the attempts in a sheet, containing the relevant information about these configurations, which helped us to find the right combination for the situations.

The model we developed reached $80.75 \%$ precision for the identification of sunspots. It can identify and label most of the sunspots in a magnetogram.

For sunspots classification, the model we developed reached an accuracy of $88 \%$. It can classify sunspot according to the magnetic classes it belongs.
In addition, we developed a Python program, which integrates the two models. The program receives a magnetogram, identifies the sunspots with the Object Detection model, and uses the sunspots as the input for the Classification model. At the end of the execution, the program saves the initial magnetogram with the sunspots outlined in red with their classifications. Figure 1 illustrates the results of the execution.

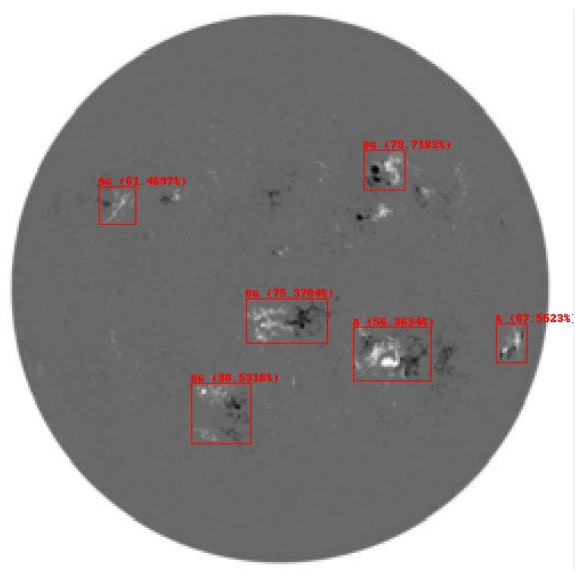

Figure 1. Results of the execution.

\section{Conclusions}

The program we developed reached the primary goal of this project, i.e., the correct identification and classification of sunspots in solar magnetograms, with an accuracy greater than $80 \%$. This program will help to forecast and to understand solar flares.

\section{Acknowledgement}

The authors acknowledge the CNPq, for the financial support of this project, and the AWS, which provided the cloud computing resources for this research.

[1] GRADVOHL, A. L. S \& FERNANDES, M. E. R. (2017). Samples of solar flares classes, active regions and time of occurrence (Version 0.1) [Dataset]. Available at: http://doi.org/10.5281/ zenodo.1048995. Access on June 29, 2019.

[2] NVIDIA DIGITS. Interactive Deep Learning GPU Training System. NVIDIA Corporation, [2016]. Available at: https://developer.nvidia.com/ digits. Access on June 29, 2019. 